\title{
CONFORMAL MODULI OF SYMMETRIC CIRCULAR QUADRILATERALS WITH CUSPS*
}

\author{
HARRI HAKULA ${ }^{\dagger}$, SEMEN NASYROV $^{\ddagger}$, AND MATTI VUORINEN $§$
}

\begin{abstract}
We investigate moduli of planar circular quadrilaterals that are symmetric with respect to both coordinate axes. First we develop an analytic approach that reduces this problem to ODEs and then devise a numerical method to find out the accessory parameters. This method uses the Schwarz equation to determine a conformal mapping of the unit disk onto a given circular quadrilateral. We also give an example of a circular quadrilateral for which the value of the conformal modulus can be found in analytic form. This example is used to validate the numeric calculations. We also apply another method, the so called hpFEM, for the numerical calculation of the moduli. These two different approaches provide results agreeing with high accuracy.
\end{abstract}

Key words. conformal capacity, conformal modulus, quadrilateral modulus, $h p$-FEM, numerical conformal mapping

AMS subject classifications. 65E05, 31A15, 30C85

1. Introduction. A planar quadrilateral is a Jordan domain $Q$ in the complex plane with four fixed points $z_{1}, z_{2}, z_{3}, z_{4}$ on its boundary; we call them the vertices of the quadrilateral and assume that they define a positive orientation. If we need to specify the vertices of a quadrilateral, then we write $Q=\left(Q ; z_{1}, z_{2}, z_{3}, z_{4}\right)$. As is well-known, there is a conformal mapping $g$ of $Q$ onto a rectangle $\Pi=(\Pi ; 1,1+h i, h i, 0), h>0$, such that the vertices of $Q$ correspond to the vertices of $\Pi$. The value $h$ does not depend on $g$; it is called the conformal modulus of $Q$,

$$
\operatorname{Mod}(Q):=h .
$$

Another method, due to L.V. Ahlfors [1, Thm 4.5, p. 63], to find the modulus is to solve the following Dirichlet-Neumann boundary value problem for the Laplace equation. Consider a planar quadrilateral $Q=\left(Q ; z_{1}, z_{2}, z_{3}, z_{4}\right)$ with the boundary $\partial Q=\cup_{k=1}^{4} \partial Q_{k}$; all the four boundary arcs are assumed to be non-degenerate. The problem is

$$
\left\{\begin{array}{rlrl}
\Delta u & =0, & & \text { on } Q, \\
u=1, & & \text { on } \partial Q_{1}=\left(z_{1}, z_{2}\right), \\
u=0, & & \text { on } \partial Q_{3}=\left(z_{3}, z_{4}\right), \\
\partial u / \partial n=0, & & \text { on } \partial Q_{2}=\left(z_{2}, z_{3}\right), \\
\partial u / \partial n=0, & & \text { on } \partial Q_{4}=\left(z_{4}, z_{1}\right) .
\end{array}\right.
$$

If we find a solution function $u$ to the above $Q$-problem, then the modulus can be computed in terms of $u$ as $\iint_{Q}|\nabla u|^{2} d x d y$. We will make use of both the above two formulations for finding the modulus. The modulus of a quadrilateral is closely related to the notion of the conformal capacity of a condenser. A condenser in the plane is a pair $(G, E)$, where $G$ is a domain in the plane and $E$ is its compact subset and its capacity is [23]

$$
\inf \int_{Q}|\nabla u|^{2} d x d y
$$

* Received February 6, 2021. Accepted May 16, 2021. Published online on May 30, 2021. Recommended by Tom De Lillo. The work of the second author is supported by the development program of the Volga Region Mathematical Center (agreement no. 075-02-2021-1393).

${ }^{\dagger}$ Aalto University, Institute of Mathematics, P.O. Box 11100, FI-00076 Aalto, Finland (harri.hakula@aalto.fi)

${ }^{\ddagger}$ Kazan Federal University, Kazan, Russia (semen. nasyrov@yandex.ru).

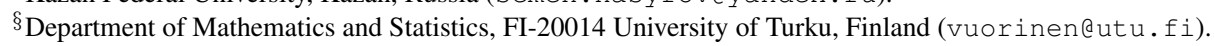




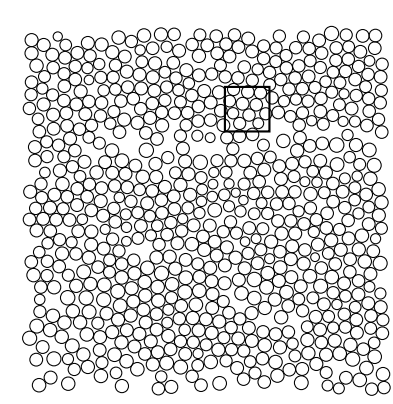

(a) Map of detected fibers with detail area.

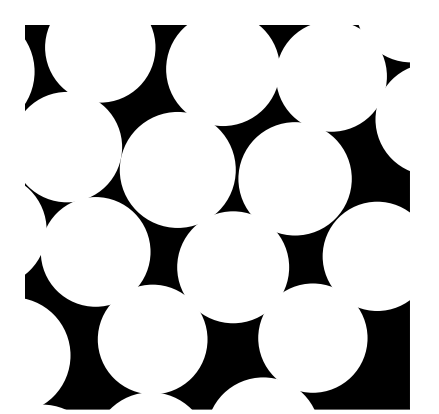

(b) Detail area with negative colours.

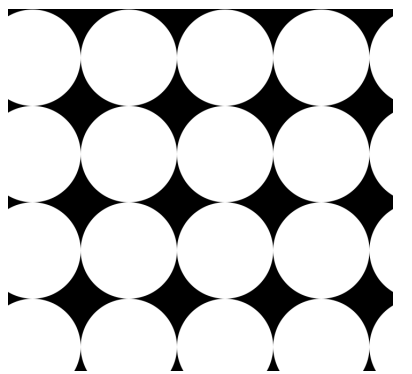

(c) Detail area after homogenization.

FIGURE 1.1. Circular polygons in applications: carbon fiber modelling. (a) Map of the measured locations of the fibres within the resin. (b) As the fibres touch, that is, there is contact, the planar intersections of the cavities form circular n-gons. (c) After homogenization, the cavities are circular quadrilaterals. Data courtesy of I. Babuška, UT Austin.

where the infimum is taken over the class of all nonnegative $C^{\infty}(G)$-functions with compact support in $G$ and $u(x) \geq 1$ for all $x \in E$.

The investigation of conformal moduli of quadrilaterals plays an important role in geometric function theory. The method of conformal moduli is a powerful tool in the theory of quasiconformal mappings in the plane and in multidimensional spaces; see [1, 2, 6, 23, 32, 35, 38]. For instance, many classical extremal problems of geometric function theory are related to moduli of quadrilaterals or capacities of condensers [6, 23, 32].

We note that conformal moduli of quadrilaterals are closely connected with those of doubly-connected planar domains. Indeed, all smooth enough doubly-connected domains can be subdivided into two quadrilaterals. In recent years, a lot of attention has been paid to the numerical computation of conformal moduli of some classes of quadrilaterals such as those associated with polygonal domains or domains bounded by circular arcs [10, 21, 26, 27, 36, 37, 41].

We investigate moduli of circular quadrilaterals bounded by four circular arcs. Naturally, the vertices of such quadrilaterals are the intersection points of the arcs. In addition, we will assume that the quadrilaterals are symmetric with respect to the real and imaginary axes, that they have zero inner angles at the vertices, and that all vertices are on the unit circle. However, these circular arcs need not be perpendicular to the unit circle. We also include curvilinear $n$-gons in our examples.

The modelling of carbon fibers induces computational domains that are rich in such domains [7]. In Figure 1.1 a map of measured fiber locations is shown with a detailed image highlighting the domains bounded by the aforementioned circular arcs. Notice that due to measurement tolerances it would be correct to assume that all sufficiently small gaps could be modelled as closed, i.e., neighbouring fibers touch each other. In fact, in order to avoid cusps, in [7] a minimum distance between fibers was imposed.

Using domain-specific discretizations of computational domains as opposed to traditional triangulations is one of the most active areas for numerical methods for partial differential equations. In particular, we want to mention the virtual element method [12] and the cut finite element method [18]. In our context, of particular interest is the contruction of finite elements on curvilinear polygons or $n$-gons [5]. Constructing quadrature rules for such elements is a challenge, and employing conformal mappings is an intriguing option yet to be fully examined. 


\section{ETNA}

Kent State University and

Johann Radon Institute (RICAM)

Our main goal is to develop numerical methods for calculating conformal moduli of the kind of circular quadrilaterals and $n$-gons mentioned above with a precision as high as possible. Both analytic and purely numerical methods are included in this study.

The analytic method (Section 2) uses conformal mappings of the unit disk onto circular quadrilaterals and their Schwarzian derivatives. This method is classical; it is used in many papers including recent ones. Here we should mention the articles [15, 16, 17, 34] concerning the usage of elliptic functions and a spectral Sturm-Liouville problem. The numerical method reduces to a solution of a pair of ordinary differential equations (ODE). In Section 2.3, making use of the Riemann-Schwarz symmetry principle, we construct a circular quadrilateral whose modulus can be determined in analytic form. We use this example to test the accuracy of the developed numerical methods.

The purely numerical method (Section 3 ) is based on the $h p$-finite element method (FEM) implemented by the first author and previously tested in [29,31]. In contrast to the first method, the moduli are now computed via potentials of the associated Dirichlet-Neumann problems. The $h p$-FEM results are paired with respective a posteriori error estimates supporting our high confidence in the accuracy of both methods studied here. Two error estimators are considered: the $h p$-FEM a posteriori error estimate based on the auxiliary space methods and the physics-based reciprocal error [28, 29]. Convergence for the latter, while general, is only a necessary condition, and thus it should always be used in connection with other error estimators. The challenges caused by the zero inner angles are well-known. We deal with this difficulty using geometric mesh grading and a control of the order of the polynomial approximation.

The two approaches are compared for a parametrized set of circular quadrilaterals in the form of graphics and tables. The results are in excellent agreement with the analytic results and support our stated goal of being as accurate as possible. The $n$-gon test is carried out with the $h p$-version only. In all cases, exponential convergence is achieved with the $h p$-version at the predicted rates [40]. These observations are supported by both types of $h p$-error estimators. We draw our conclusions in Section 4 and include a sample implementation of the analytic method in Appendix A.

Several authors have studied various topics about conformal mappings of domains with circular arc boundaries. The difficulties encountered already in the case of nonsymmetric quadrilaterals are pointed out in [22, Section 4.10]. The interested reader might want to look at $[10,11,14,17,21,36]$.

\section{The method of conformal mappings.}

2.1. Circular quadrilaterals and the Schwarz equation. First we recall some classical results about conformal mappings of canonical domains onto circular polygons. Let $D$ be a Jordan domain, and let its boundary consist of $n$ circular $\operatorname{arcs} A_{k-1} A_{k}, 1 \leq k \leq n$, with $A_{0}=A_{n}$. We will name $D$ a circular polygon, and the points $A_{k}$ are called the vertices of $D$. Denote by $\alpha_{k} \pi, 0 \leq \alpha_{k} \leq 2$, the inner angle of $D$ at the vertex $A_{k}$.

By definition, the Schwarzian derivative of a meromorphic function $f$ is the expression

$$
S_{f}(z)=\left(\frac{f^{\prime \prime}(z)}{f^{\prime}(z)}\right)^{\prime}-\frac{1}{2}\left(\frac{f^{\prime \prime}(z)}{f^{\prime}(z)}\right)^{2} .
$$

Let now $f$ be a conformal mapping of the unit disk $U:=\{|z|<1\}$ onto $D$, and denote by $a_{k}$ the preimage of $A_{k}$ under the map $f$ lying on the unit circle $\partial U:=\{|z|=1\}$. The 
following theorem describes the form of the Schwarzian derivative of $f$; see, e.g., [25, Ch. 3, $\S 1],[45, \S 12]$.

THEOREM 2.1. The Schwarzian derivative of the conformal mapping $f$ of $U$ onto the circular polygon $D$ has the form

$$
S_{f}(z)=\sum_{k=1}^{n} \frac{\left(1-\alpha_{k}^{2}\right) / 2}{\left(z-a_{k}\right)^{2}}+\frac{C_{k}}{z-a_{k}} .
$$

Here the parameters $C_{k}$ are some complex numbers satisfying the relations

$$
\begin{aligned}
\sum_{k=1}^{n} C_{k} & =0, \\
\frac{1}{2} \sum_{k=1}^{n}\left(1-\alpha_{k}^{2}\right)+\sum_{k=1}^{n} C_{k} a_{k} & =0, \\
\sum_{k=1}^{n}\left(1-\alpha_{k}^{2}\right) a_{k}+\sum_{k=1}^{n} C_{k} a_{k}^{2} & =0 .
\end{aligned}
$$

From Theorem 2.1 we see that the expression (2.1) for the Schwarzian derivative of $f$ contains $n$ unknown constants (or so-called accessory parameters) $C_{k}$. Finding these constants is very complicated. The problem of determining a function by its given Schwarzian derivative is well known; many papers are devoted to this investigation. Various methods are used to study the problem such as the parametric method [4, 9, 20, 33, 35], boundary value problems [19, 42, 43, 44], Polubarinova-Kochina's method [13], the method of asymptotic integration [45], and others. Some of these references point out that the problem of accessory parameters is very important for investigations in fluid mechanics, especially in filtration theory.

If we know the values of $C_{k}$, then the problem of finding $f$ is reduced to solving the nonlinear third-order differential equation

$$
\left(\frac{f^{\prime \prime}(z)}{f^{\prime}(z)}\right)^{\prime}-\frac{1}{2}\left(\frac{f^{\prime \prime}(z)}{f^{\prime}(z)}\right)^{2}=\sum_{k=1}^{n} \frac{\left(1-\alpha_{k}^{2}\right) / 2}{\left(z-a_{k}\right)^{2}}+\frac{C_{k}}{z-a_{k}} .
$$

The following theorem gives a connection between this problem and the integration of linear second-order differential equation; see, e.g., [24, Ch. VI], [44, 45].

THEOREM 2.2. Let the Schwarzian derivative $S_{f}$ of $f$ be given. Then $f$ is defined by $S_{f}$ up to a Möbius transformation. The general solution of the problem is given by the formula

$$
f(z)=\frac{u(z)}{v(z)} .
$$

Here $u$ and $v$ are arbitrary linear independent solutions of the equation

$$
h^{\prime \prime}(z)+(1 / 2) S_{f}(z) h(z)=0 .
$$

REMARK 2.3. Assume that we seek an odd solution to the problem in a domain $G$ containing the origin and being symmetric with respect to the origin. Let $S_{f}(z)$ be an even function in $G$. Then we can take $u$ and $v$ as odd and even solutions of equation (2.2) in $G$. Therefore, we find $u$ and $v$ as solutions to (2.2) with the following conditions:

$$
u(0)=0, \quad u^{\prime}(0)=C \neq 0, \quad v(0)=1, \quad v^{\prime}(0)=0 .
$$




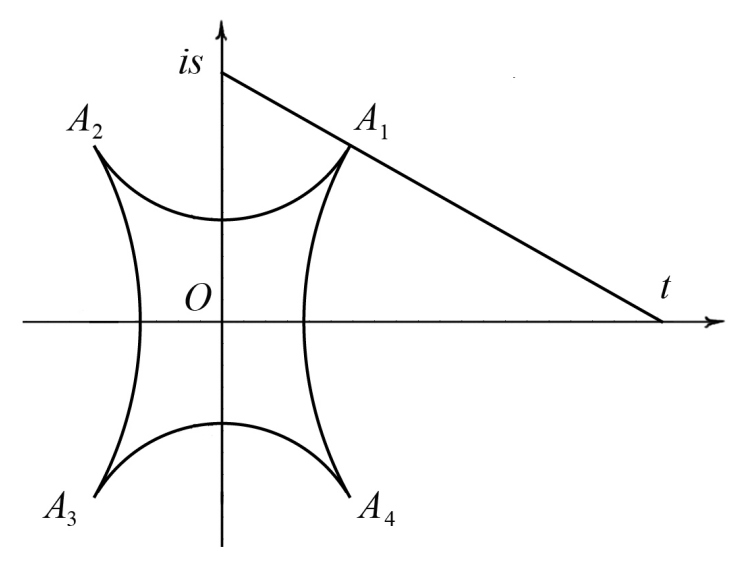

FIGURE 2.1. Symmetric circular quadrilateral with zero angles.

2.2. Conformal mapping of symmetric circular quadrilaterals. We now apply Theorems 2.1 and 2.2 in a special case. Let $A_{1} A_{2} A_{3} A_{4}$ be a circular quadrilateral with zero inner angles, symmetric with respect to both axes. Let the centers of the circles containing the circular arcs $A_{k-1} A_{k}$ be at the points $\pm t, \pm i s$, where $t, s>0$. We also assume that at the points $A_{k}$, the circles touch each other externally; see Figure 2.1. Denote the radii of the circles centered at $\pm t$ and $\pm i s$ by $r_{1}$ and $r_{2}$. Then, by Pythagoras' theorem, $t^{2}+s^{2}=\left(r_{1}+r_{2}\right)^{2}$.

Denote by $f$ the conformal mapping of the unit disk onto $A_{1} A_{2} A_{3} A_{4}$. Because of the symmetry of the quadrilateral with respect to the coordinate axes and the Riemann-Schwarz symmetry principle, we may assume without loss of generality that

$$
f(\bar{z})=\overline{f(z)}, \quad f(-z)=-f(z) .
$$

Therefore, the preimages $a_{k}$ of the vertices $A_{k}$ are symmetric with respect to the axes, i.e., we can put

$$
a_{1}=e^{i \beta}, \quad a_{2}=-e^{-i \beta}, \quad a_{3}=-e^{i \beta}, \quad \text { and } \quad a_{4}=e^{-i \beta}, \quad 0<\beta<\pi / 2 .
$$

Because all the angles of the quadrilateral equal zero, we have $\alpha_{k}=0,1 \leq k \leq 4$. Then, by Theorem 2.1, the Schwarzian derivative of $f$ has the form

$$
\begin{aligned}
S_{f}(z)=\frac{1}{2}\left[\frac{1}{\left(z-e^{i \beta}\right)^{2}}+\frac{1}{\left(z+e^{-i \beta}\right)^{2}}\right. & \left.+\frac{1}{\left(z+e^{i \beta}\right)^{2}}+\frac{1}{\left(z-e^{-i \beta}\right)^{2}}\right] \\
+ & {\left[\frac{C_{1}}{z-e^{i \beta}}+\frac{C_{2}}{z+e^{-i \beta}}+\frac{C_{3}}{z+e^{i \beta}}+\frac{C_{4}}{z-e^{-i \beta}}\right] . }
\end{aligned}
$$

Here, the constants $C_{k}, 1 \leq k \leq 4$, satisfy

$$
\begin{aligned}
C_{1}+C_{2}+C_{3}+C_{4} & =0 \\
e^{i \beta} C_{1}-e^{-i \beta} C_{2}-e^{i \beta} C_{3}+e^{-i \beta} C_{4} & =-2, \\
e^{i 2 \beta} C_{1}+e^{-i 2 \beta} C_{2}+e^{i 2 \beta} C_{3}+e^{-i 2 \beta} C_{4} & =0 .
\end{aligned}
$$

From the first and third equations of the system we obtain $C_{1}+C_{3}=0, C_{2}+C_{4}=0$, hence, $C_{3}=-C_{1}, C_{4}=-C_{2}$. From (2.3) it follows that $C_{4}=\overline{C_{1}}$. Moreover, (2.5) implies $\operatorname{Re}\left[e^{i \beta} C_{1}\right]=-1 / 2$. 
Denote $\operatorname{Im}\left[e^{i \beta} C_{1}\right]=\delta$. Then

$e^{i \beta} C_{1}=-1 / 2+i \delta, \quad e^{-i \beta} C_{2}=1 / 2+i \delta, \quad e^{i \beta} C_{3}=1 / 2-i \delta, \quad e^{-i \beta} C_{4}=-1 / 2-i \delta$.

After simple transformations we obtain (see also [39])

$$
\frac{1}{2} S_{f}(z)=\frac{e^{i 2 \beta}}{\left(z^{2}-e^{i 2 \beta}\right)^{2}}+\frac{e^{-i 2 \beta}}{\left(z^{2}-e^{-i 2 \beta}\right)^{2}}-\frac{\gamma}{\left(z^{2}-e^{i 2 \beta}\right)\left(z^{2}-e^{-i 2 \beta}\right)},
$$

where $\gamma=2 \delta \sin 2 \beta \in \mathbb{R}$. By Theorem 2.2, taking into account (2.3), we represent $f$ in the form

$$
f(z)=C \frac{u(z)}{v(z)}, \quad C>0
$$

where $C$ is a constant and

$$
u(z)=z+\sum_{k=2}^{\infty} a_{k} z^{k}+\ldots \quad \text { and } \quad v(z)=1+\sum_{k=1}^{\infty} b_{k} z^{k}
$$

are linearly independent solutions to the ODE (2.2). Taking into account that, by (2.3), $f$ is odd and $S_{f}$ is an even function, we conclude with the help of Remark 2.3 that $u(z)$ is odd and $v(z)$ is even. Thus,

$$
\begin{array}{ll}
u^{\prime \prime}(z)+(1 / 2) S_{f}(z) u(z)=0, & u(0)=0, u^{\prime}(0)=1 \\
v^{\prime \prime}(z)+(1 / 2) S_{f}(z) v(z)=0, & v(0)=1, v^{\prime}(0)=0 .
\end{array}
$$

Therefore, we have the following result:

THEOREM 2.4. Let $f$ be the conformal mapping of the unit disk onto a symmetric circular quadrilateral $Q$ with zero angles such that the points $\pm e^{ \pm i \beta}, 0<\beta<\pi / 2$, correspond to the vertices of $Q$. Then the Schwarzian derivative of $f$ is a rational function expressed by (2.6) with some real $\gamma$, $f$ has the form (2.7), where $C$ is a positive constant, and the functions $u$ and $v$ are solutions of the problems (2.8) and (2.9).

We should note that the result for the form of the Schwarzian derivative is actually obtained in [39, Appendix] for a more general case. Here we focus on the case of zero angles.

The equations (2.8) and (2.9) can be used to find the values of $\beta$ and $\gamma$, corresponding to a given circular quadrilateral $Q=A_{1} A_{2} A_{3} A_{4}$. If we fix some values of the parameters $\beta$ and $\gamma$ and solve the boundary problems for the ODEs, then we find the mapping $f(z)=C u(z) / v(z)$ up to a factor $C \neq 0$. The obtained function $f(z)=f(z ; \beta, \gamma)$ maps the unit disk onto a symmetric circular quadrilateral with zero inner angles, possibly non-uniquely. It is evident that for a given symmetric circular quadrilateral $Q$, there is a unique pair $(\beta, \gamma)$ such that $f(z ; \beta, \gamma)$, with an appropriate value of $C$, maps the unit disk onto $Q$.

Therefore, the main problem is to find such a pair $(\beta, \gamma)$ for a given $Q$. We note that the parameter $\beta$ has a very simple geometric meaning. Finding $\beta$ is equivalent to finding the conformal modulus of $Q$. Actually, because of the property of conformal invariance, the modulus of $Q$ is equal to the modulus of the unit disk with vertices (2.4), which depends only on $\beta$. The parameter $\gamma$ has no simple geometric meaning but it also affects the geometry of $Q$.

To find $(\beta, \gamma)$ numerically, we seek $u\left(e^{i \theta}\right)$ and $v\left(e^{i \theta}\right)$ as solutions of the following boundary value problems for ODEs:

$$
\begin{aligned}
& u^{\prime \prime}\left(r e^{i \theta}\right)+\frac{e^{i 2 \theta}}{2} S_{f}\left(r e^{i \theta}\right) u\left(r e^{i \theta}\right)=0, \quad 0 \leq r \leq 1, \quad u(0)=0, u^{\prime}(0)=e^{i \theta}, \\
& v^{\prime \prime}\left(r e^{i \theta}\right)+\frac{e^{i 2 \theta}}{2} S_{f}\left(r e^{i \theta}\right) v\left(r e^{i \theta}\right)=0, \quad 0 \leq r \leq 1, \quad v(0)=1, v^{\prime}(0)=0,
\end{aligned}
$$


and determine $f\left(e^{i \theta}\right)=C u\left(e^{i \theta}\right) / v\left(e^{i \theta}\right)$. Then we find the values $T=f(1), S=f(i) / i$, and the values of the radii $R_{1}$ and $R_{2}$ of the circles containing the circular $\operatorname{arcs} f\left(e^{i \theta}\right)$, $-\beta<\theta<\beta$, and $f\left(e^{i \theta}\right), \beta<\theta<\pi-\beta$. All these values depend on the parameters $\beta$ and $\gamma$. Then we compare the ratios $S / T$ and $R_{2} / R_{1}$ with the given ones, $s / t$ and $r_{2} / r_{1}$. Therefore, we have two equations to determine $\beta$ and $\gamma$ :

$$
\frac{S(\beta, \gamma)}{T(\beta, \gamma)}=\frac{s}{t}, \quad \frac{R_{2}(\beta, \gamma)}{R_{1}(\beta, \gamma)}=\frac{r_{2}}{r_{1}} .
$$

This system has a unique solution, and the obtained value of $\beta$ enables us to find the modulus of $Q$.

Because our main goal is to determine the conformal modulus of $Q$ by solving the system (2.12) and because the ratios $R / T$ and $R_{2} / R_{1}$ do not change under homotheties, we will often assume below that the constant $C$ in (2.7) equals 1 .

2.3. Example of circular quadrilaterals with exactly known modulus. Unfortunately, there are very few examples of concrete circular quadrilaterals with exactly known values of the conformal modulus. In this section, with the help of the Riemann-Schwarz symmetry principle and the Schwarz-Christoffel formula, we give an example of this type.

Consider the circular quadrilateral $Q, Q \subset U$, with vertices lying on the unit circle $\partial U$ at the points

$$
A_{1}=e^{i \alpha}, A_{2}=-e^{-i \alpha}, A_{3}=-e^{i \alpha}, \text { and } A_{4}=e^{-i \alpha}, \quad \alpha=\arcsin (1 / \sqrt{3}) .
$$

Let the boundary arcs of $Q$ be orthogonal to the unit circle. The Möbius transformation

$$
\omega=(i \cot \alpha) \frac{e^{-i \alpha}-z}{e^{-i \alpha}+z}
$$

maps $Q$ conformally onto the circular quadrilateral $D$ lying in the upper half-plane of the variable $\omega$, bounded by two rays $\{\operatorname{Re} \omega=-2, \operatorname{Im} \omega \geq 0\}$ and $\{\operatorname{Re} \omega=1, \operatorname{Im} \omega \geq 0\}$ and two semicircles $\{|\omega+1|=1, \operatorname{Im} \omega \geq 0\}$ and $\{|\omega-1 / 2|=1 / 2, \operatorname{Im} \omega \geq 0\}$; see Figure 2.2(A). For convenience and brevity of notation, we use the same notations for boundary points corresponding to each other in different complex planes under the applied conformal mappings. Denote by $D_{1}$ the subdomain of $D$ lying in the strip $\{-1<\operatorname{Re} \omega<0\}$. Let $F$ be the conformal map of $D_{1}$ onto the half-strip $G_{1}=\{-1<\varphi<0, \psi>0\}$ in the $w$-plane $(w=\varphi+i \psi)$ such that $F(-1+i)=-1, F(0)=0$, and $F(\infty)=\infty$. Applying the Riemann-Schwarz symmetry principle, we extend the mapping $F$ to the domains $D_{2}$ and $D_{3}$, symmetric to $D_{1}$ with respect to the lines $\operatorname{Re} \omega=-1$ and $\operatorname{Re} \omega=0$. Then the extended mapping, for which we keep the same notation $F$, maps the union of the domains $D_{1} \cup D_{2} \cup D_{3}$ (supplemented with their common boundary arcs) onto the strip $\{-2<\varphi<1\}$ consisting of three half-strips, $G_{1}, G_{2}$, and $G_{3}$; see Figure 2.2. At last, we can extend $F$ by symmetry to the domain $D_{4}$, symmetric to $D_{3}$ with respect to the boundary arc $A_{4} B$, lying on the unit circle $\{|w|=1\}$. The extended function maps $D_{4}$ conformally onto the half-strip $G_{4}$ symmetric to $G_{4}$ with respect to the real axis. As a result, we conclude that the domain $D$ is conformally equivalent to the strip-shaped domain $G$ glued from the half-strips $G_{k}, 1 \leq k \leq 4$, along their common boundary segments.

Let us conformally map the upper half-plane in the $\zeta$-plane onto $G$ such that the points $-1 / \lambda,-1,1$, and $1 / \lambda(\lambda>1)$ correspond to $A_{2}, A_{3}, A_{4}$, and $A_{1}$. The desired mapping is given by the Schwarz-Christoffel integral

$$
G(\zeta)=c \int_{1}^{\zeta} \sqrt{\frac{1-t}{1+t}} \frac{d t}{1-\lambda^{2} t^{2}}
$$




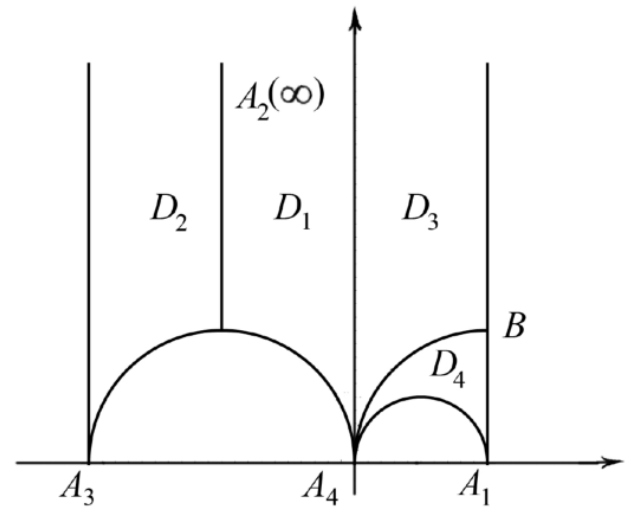

(A)

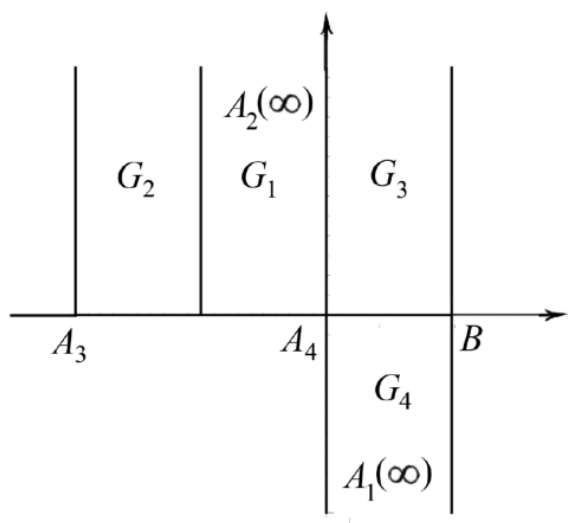

(B)

FIGURE 2.2. (A) Circular quadrilateral in the upper half-plane. (B) its strip-shaped image.

with some constant $c>0$. In a neighborhood of $\zeta=1 / \lambda$ we have

$$
G(\zeta) \sim i \frac{c}{2 \lambda} \sqrt{\frac{1-\lambda}{1+\lambda}} \log (\zeta-1 / \lambda) .
$$

In a similar way, as $\zeta \rightarrow-1 / \lambda$, we have

$$
G(\zeta) \sim-i \frac{c}{2 \lambda} \sqrt{\frac{1+\lambda}{1-\lambda}} \log (\zeta+1 / \lambda) .
$$

We recall that the function $\log (\zeta)$ maps the upper half of a sufficiently small neighborhood of the origin conformally onto a half-strip-like domain of width $\pi$. Taking into account the values of the widths of the half-strip parts of $G$ and the asymptotics (2.13), (2.14), we obtain

$$
\frac{\pi c}{2 \lambda} \sqrt{\frac{1-\lambda}{1+\lambda}}=1, \quad \frac{\pi c}{2 \lambda} \sqrt{\frac{1+\lambda}{1-\lambda}}=3 .
$$

Therefore, $(1+\lambda) /(1-\lambda)=3$ and $\lambda=1 / 2$.

Now we can find the value of the conformal modulus of $Q$. Because of the invariance of the modulus under conformal mappings, we see that $\operatorname{Mod}(Q)$ is equal to the modulus of the quadrilateral that is the upper half-plane with vertices $\pm 1, \pm 1 / \lambda$. Therefore, it can be computed via elliptic integrals; see, e.g., [3, 6]. Namely,

$$
(\operatorname{Mod}(Q))^{-1}=\frac{2 K(\lambda)}{K\left(\lambda^{\prime}\right)}
$$

where

$$
K(\lambda)=\int_{0}^{1} \frac{d t}{\sqrt{\left(1-t^{2}\right)\left(1-\lambda^{2} t^{2}\right)}}
$$

is the complete elliptic integral of the first kind and $\lambda^{\prime}=\sqrt{1-\lambda^{2}}=\sqrt{3} / 2$. At last, we obtain

$$
\operatorname{Mod}(Q)=\frac{K(\sqrt{3} / 2)}{2 K(1 / 2)}=0.6396307855855 \ldots
$$




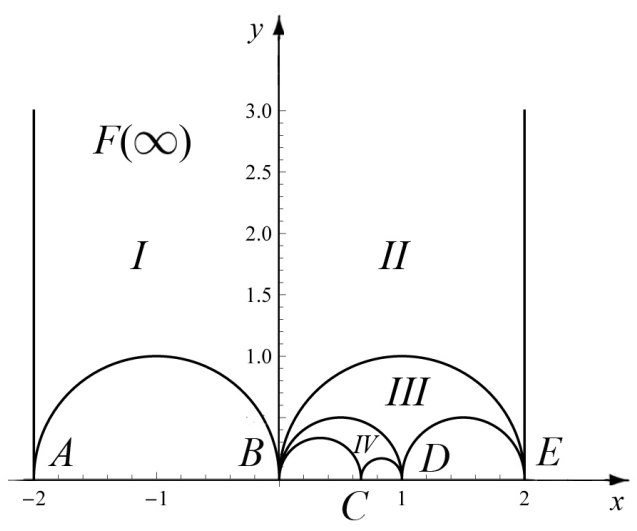

(A)

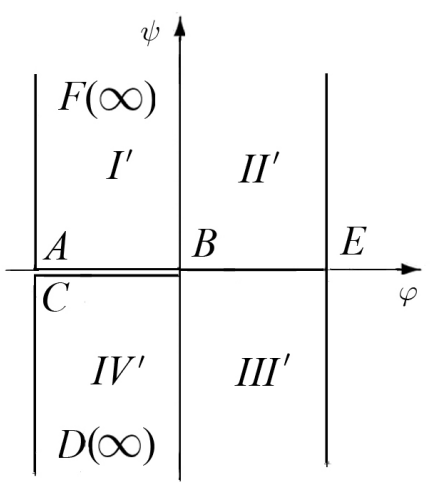

(B)

FIGURE 2.3. (A) Circular hexagon in the upper half-plane. (B) its polygonal image.

Now we will find the Schwarzian derivative of the conformal mapping of the unit disk onto $Q$. As we noted in Section 2.2, it has the form (2.6). The cross-ratio of a quadruple

$$
\left(z_{1}, z_{2} ; z_{3}, z_{4}\right)=\frac{\left(z_{3}-z_{1}\right)\left(z_{4}-z_{2}\right)}{\left(z_{3}-z_{2}\right)\left(z_{4}-z_{1}\right)}
$$

is invariant under Möbius transformations. Comparing the cross-ratios for vertices of the two quadrilaterals, the first one of which is the unit disk with vertices $\pm e^{ \pm i \beta}$ and the second one is the upper half-plane with vertices \pm 1 and $\pm 1 / \lambda$, we obtain

$$
\sin \beta=\frac{1-\lambda}{1+\lambda}=\frac{1}{3},
$$

therefore $\beta=\arcsin (1 / 3)$. A careful analysis of the Schwarzian derivative in small neighborhoods of the points $\pm e^{ \pm i \beta}$ shows that, in the considered case, the parameter $\gamma$ in (2.6) equals $2 / 3$.

2.4. Conformal mapping of circular $\boldsymbol{n}$-gons. It is of interest to consider circular $n$ gons with $n>4$ and to compute moduli of quadrilaterals which are obtained from them after fixing four of their vertices.

Here we give some examples of circular $n$-gons with zero angles and known conformal moduli of quadrilaterals constructed based on these $n$-gons. They can be also used for testing the error of the $h p$-FEM for finding conformal moduli as shown below in Section 3.

EXAMPLE 2.5. In the $z$-plane $z=x+i y$ we consider a circular hexagon $H$ with zero angles. The hexagon is obtained from the half-strip $\{-2<x<2, y>0\}$ by removing points lying in the disks $\left\{(x+1)^{2}+y^{2} \leq 1\right\},\left\{(x-1 / 3)^{2}+y^{2} \leq 1 / 9\right\},\left\{(x-5 / 6)^{2}+y^{2} \leq 1 / 36\right\}$, and $\left\{(x-3 / 2)^{2}+y^{2} \leq 1 / 4\right\}$. It has vertices at the points (Figure 2.3(A))

$$
A(-2,0), \quad B(0,0), \quad C(2 / 3,0), \quad D(1,0), \quad E(2,0), \quad \text { and } \quad F(\infty) \text {. }
$$

Let us map the circular triangle $I:=\{-2<x<0, y>0\} \backslash\left\{(x+1)^{2}+y^{2} \leq 1\right\}$ conformally onto the half-strip $I^{\prime}:=\{-\pi / 2<\varphi<0, \psi>0\}$ in the $w$-plane $w=\varphi+i \psi$. Applying three times the Riemann-Schwarz symmetry principle, we extend the mapping step by step to the circular triangles designated by $I I, I I I$, and $I V$ in Figure 2.3(A). The extended function $f$ maps the triangles onto the half-strips $I I^{\prime}, I I I^{\prime}$, and $I V^{\prime}$; see Figure 2.3(B). 
Therefore, $f$ maps $H$ conformally onto the strip $\{|\varphi|<\pi / 2\}$ with the slit along the segment $\{-\pi / 2<\varphi<0, \psi=0\}$. Denote this domain by $\Omega$. The function $\omega=g(w):=$ $2 \sin w-1$ maps $\Omega$ conformally onto the $\omega$-plane $\omega=\xi+i \eta$ with two slits along the rays $\{\xi \leq-1, \eta=0\}$ and $\{\xi \geq 1, \eta=0\}$. Then we apply the function inverse to the Joukowsky function, $\sigma=h(\omega)=\omega+\sqrt{\omega^{2}-1}$, with an appropriate choice of the regular branch of the square root. The composition $h \circ g \circ f$ maps the hexagon $H$ conformally onto the upper half-plane with the following correspondence of points:

$$
A \mapsto-(3+2 \sqrt{2}), \quad B \mapsto-1, \quad C \mapsto-(3-2 \sqrt{2}), \quad D \mapsto 0, \quad E \mapsto 1, \quad F \mapsto \infty
$$

Therefore, we have the following result:

The hexagon $H$ is conformally equivalent to the upper half-plane with the correspondence of points given by (2.15).

Because the conformal modulus of the quadrilateral that is the upper half-plane with four fixed vertices on the real axis is well-known, we can fix any four of the six vertices and easily compute the modulus of the obtained quadrilateral.

REMARK 2.6. In Section 3 we use this example to verify the accuracy of the $h p$-FEM for determining conformal moduli of circular $n$-gons. This method needs a calculation of double integrals over a given $n$-gon. Therefore, it is better to apply it in a bounded domain. Since the modulus is a conformal invariant, we can consider, instead of the unbounded hexagon $H$, its conformal image under the Möbius transformation

$$
w=T(z)=\frac{4-(1-3 i) z}{4-(1+3 i) z} .
$$

This transformation maps the upper half-plane onto the unit disk, and $Q$ corresponds to the hexagon bounded by circular arcs orthogonal to the unit circle. Moreover, there is the following correspondence between the vertices of $Q$ and their images:

$$
A \mapsto-i, B \mapsto 1, C \mapsto \frac{8+15 i}{17}, D \mapsto i, \quad E \mapsto \frac{-4+3 i}{5}, F \mapsto \frac{-4-3 i}{5} .
$$

The obtained hexagon is considered in Section 3.3.3; see Figure 3.2.

EXAMPLE 2.7. Given $n \geq 4$, consider the circular $n$-gon $P_{n}$ which is obtained from the half-strip $\{0<x<2(n-2), y>0\}$ by removing the disks $D_{k}:=\left\{(x-(2 k-1))^{2}+y^{2} \leq 1\right\}$, $1 \leq k \leq n-2$. It has zero angles and vertices at the points $0,2,4, \ldots, 2 n-2, \infty$. We map the triangle $\{0<x<2, y>0\} \backslash D_{1}$ onto the half-strip $\{0<\varphi<2, \psi>0\}$ and extend the mapping by symmetry to a conformal mapping of the $n$-gon onto the half-strip $S:=\{0<\varphi<2(n-2), \psi>0\}$. Then we map $S$ onto the upper half-plane by the function $\zeta=-\cos \frac{\pi w}{2(n-2)}$ such that the vertices of $P_{n}$ are mapped to the points $-\cos \frac{\pi k}{n-2}$, $k=0,1, \ldots, n-2$, and $\infty$. As in Example 2.5, by fixing four vertices of $P_{n}$ we can find the exact value of the modulus of the obtained quadrilateral.

2.5. Numeric results. Here we give a numerical algorithm to find the values of $\beta$ and $\gamma$ in (2.6) for a given symmetric quadrilateral with zero inner angles; see Figure 2.1. For this, as indicated above, we need to solve the system (2.12).

Denote $k:=s / t, K:=r_{2} / r_{1}$. Then (2.12) has the form

$$
S(\beta, \gamma) / T(\beta, \gamma)=k, \quad R_{2}(\beta, \gamma) / R_{1}(\beta, \gamma)=K .
$$

First we describe, how, for given arbitrary $\beta$ and $\gamma$, to find the centers $T(\beta, \gamma)$ and $i S(\beta, \gamma)$ and the radii $R_{1}(\beta, \gamma)$ and $R_{2}(\beta, \gamma)$ of the circles which contain the boundary circular arcs of 


\section{ETNA}

Kent State University and

Johann Radon Institute (RICAM)

the corresponding circular polygon. We note that boundary arcs are symmetric with respect to either the real or the imaginary axis, therefore it is sufficient to determine two distinct points for each of the circles. We can find $f\left(e^{i \theta}\right)=u\left(e^{i \theta}\right) / v\left(e^{i \theta}\right)$ by solving the equations (2.10) and (2.11), where $S_{f}$ is defined by (2.6) and corresponds to the fixed values of $\beta$ and $\gamma$. Then we take two different values of $\theta$ from $[0, \beta)$, say, $\theta_{1}=0$ and $\theta_{2}=\beta / 2$, and find the values of $f\left(e^{i \theta_{1}}\right)$ and $f\left(e^{\theta_{2}}\right)$. Let $x_{1}=f\left(e^{i \theta_{1}}\right)$ and $x_{2}+i y_{2}=f\left(e^{\theta_{2}}\right)$. Next we determine

$$
T=(1 / 2)\left(x_{1}+x_{2}+y_{2}^{2} /\left(x_{2}-x_{1}\right)\right), \quad R_{1}=\left|t-x_{1}\right|,
$$

where $x_{1}=f\left(e^{i \theta_{1}}\right)$ and $x_{2}+i y_{2}=f\left(e^{\theta_{2}}\right)$. Similarly, we fix two angles in $(\beta, \pi / 2]$, say, $\theta_{3}=\pi / 4+\beta / 2$ and $\theta_{4}=\pi / 2$, and find

$$
S=(1 / 2)\left(y_{3}+y_{4}+x_{4}^{2} /\left(y_{4}-y_{3}\right)\right), \quad R_{2}=\left|s-y_{4}\right|,
$$

where $x_{3}+i y_{3}=f\left(e^{i \theta_{3}}\right), i y_{4}=f\left(e^{\theta_{4}}\right)$.

Now we describe how to solve the system (2.16). Initially, for a given fixed $\beta$, we solve the first equation from (2.16) with respect to $\gamma$. We should note that for given values of $\beta$ and $k$, a symmetric circular quadrilateral, and therefore $\gamma$, is not uniquely determined. We consider circular polygons such that the circles, containing their boundary, touch each other externally at intersection points. But there could be another circular polygon with the same values of $\beta$ and $k$ and the circles touching internally. To avoid this, first, for a given $\beta$ we need to determine the values of $\gamma, A_{\gamma}$, and $B_{\gamma}, A_{\gamma}<B_{\gamma}$, for which we obtain circular quadrilaterals with a pair of sides lying on parallel straight lines. The values $A_{\gamma}$ and $B_{\gamma}$ correspond to the conditions $x_{1}=0\left(R_{1}=\infty\right)$ and $y_{4}=0\left(R_{2}=\infty\right)$.

We find the values of $A_{\gamma}$ and $B_{\gamma}$ by the bisection method on some segment $I=\left[a_{\gamma}, b_{\gamma}\right]$. The segment $I$ must be sufficiently large and must contain $A_{\gamma}$ and $B_{\gamma}$. Using a numerical experiment, we determined that for a wide class of $\beta$ and $k$ the following values of the parameters are appropriate:

$$
a_{\gamma}=0.7-(4 / \pi) \beta, \quad b_{\gamma}=1.2-(3 / \pi) \beta .
$$

When the values of $A_{\gamma}$ and $B_{\gamma}$ are found, we determine the desired value of $\gamma=\gamma(\beta)$.

To fulfill the second equality in (2.16), we solve the equation

$$
R_{2}(\beta, \gamma(\beta)) / R_{1}(\beta, \gamma(\beta))=K,
$$

making use of the bisection method on the segment $[0, \pi / 4]$. We do not know a priori whether $\beta<\pi / 4$; this means that the modulus is less than 1 . If it turns out that the numerical value of the desired modulus is greater than 1 and therefore $\beta \geq \pi / 4$, then the bisection method on the segment $[0, \beta / 4]$ converges to the boundary value $\beta=\pi / 4$. In this case, we swap the values of $t$ and $s$, as well as $r_{1}$ and $r_{2}$, and repeat the calculations for these updated values; at the end, the found value of $\beta$ must be changed to $\pi / 2-\beta$.

For numeric calculations we use the Wolfram Mathematica software. If we want to obtain the approximate value of the modulus quickly and with accuracy about $10^{-6}$, for solving Cauchy's problems for differential equations with the help of NDSolve we can use the option PrecisionGoal $\rightarrow 15$. To find the parameters $A_{\gamma}$ and $B_{\gamma}$ with the help of the bisection method, it is sufficient to use 10 iterations, and for each of the parameters $\beta$ and $\gamma$ we used 25 iterations.

In Appendix A we provide the Mathematica code for the calculation of the conformal moduli. The input values of $t, s, r_{1}$, and $r_{2}$ (lines 1-4) match Example 2.9 below with $\alpha=\pi / 5$ and $j=3$. The output is the found values of $\operatorname{Mod}(Q), \beta$, and $\gamma$ in line 118 .

If we need higher accuracy, then we can first find the approximate values $\beta$ and $\gamma$ with accuracy $10^{-6}$. Denote them by $\beta_{0}$ and $\gamma_{0}$. Then we use the bisection method with respect to 
$\beta$ and $\gamma$ assuming that $\beta \in\left[\beta_{0}-\varepsilon, \beta_{0}+\varepsilon\right]$ and $\gamma \in\left[\gamma_{0}-\varepsilon, \gamma_{0}+\varepsilon\right]$ with a sufficiently small $\varepsilon$, say $\varepsilon=2 \cdot 10^{-6}$. Certainly, in that case, we omit the first two steps connected with finding the values of $A_{\gamma}$ and $B_{\gamma}$. To find $\beta$ and $\gamma$, we use NDSolve with the option PrecisionGoal $\rightarrow 30$ for each of the parameters. The number of iterations is 30 . With this enhanced method, the accuracy is about $10^{-10}$ to $10^{-11}$, although it needs much more computing time; a few minutes instead of $10-15$ seconds.

Now we provide some numerical results.

EXAMPLE 2.8. For $k=\sqrt{2}$ and $K=2$ we know the exact values $\beta=\arcsin (1 / 3)$ and $\gamma=2 / 3$; see Section 2.3. Using the options PrecisionGoal $->15$, WorkingPrecision $->30$ with the number of steps equaling 30 for each of the parameters, we find that the approximate values are

$$
\sin \beta=0.3333333333332441, \quad \gamma=0.666666666666788 .
$$

Therefore, the absolute error is about $1.2 \cdot 10^{-13}$.

EXAMPLE 2.9. Assume that the vertex $A_{1}$ is $e^{i \alpha}, \alpha=\pi / n, n \in \mathbb{N}, 4 \leq n \leq 8$, as in Figure 2.1. For every $n$, we consider the following five values of $t$ :

$$
t=1+0.2 j(1 / \cos \alpha-1), \quad 1 \leq j \leq 5 .
$$

Then,

$$
s=\frac{t \sin \alpha}{t-\cos \alpha}, \quad r_{1}=\left|e^{i \alpha}-t\right|, \quad \text { and } \quad r_{2}=\left|e^{i \alpha}-i s\right| .
$$

We computed the values of the conformal modulus for these 25 cases. In Table 2.1 we present the values obtained with rough accuracy, with higher accuracy, and by the $h p$-FEM discussed in Section 3. We see that for given $\theta$, the difference in the results, shown in the fourth (higher accuracy) and fifth ( $h p$-FEM variant) columns does not exceed $5 \cdot 10^{-10}$. This indicates a fairly good accuracy of the suggested methods.

We note that in Section 3.3.3, the following two cases are considered in more detail: $n=4, j=1$ (quadrilateral $Q_{1}$ ) and $n=8, j=5$; quadrilateral $Q_{2}$.

3. Moduli via potentials. The finite element method (FEM) is the standard numerical method for solving elliptic partial differential equations. Since the FEM is an energy minimization method, it is eminently suitable for problems involving the Dirichlet energy. In the context of this paper, where the focus is on domains with zero inner angles at the vertices, the $h p$-FEM variant is the most efficient one [8, 40]. With proper grading of the meshes, even with uniform polynomial order, exponential convergence can be achieved even in problems with strong corner singularities. In this section we give a brief overview of the method and our implementation [29, 31]. Of particular importance is the possibility to estimate the error in the computed quantity of interest. For quadrilaterals there exists a natural error estimate, the so-called reciprocal relation which is a necessary but not sufficient condition for convergence. However, if the reciprocal relation is coupled with a posteriori error estimates, we can trust the results with high confidence [28].

3.1. Modulus of quadrilateral and the Dirichlet integral. Let $Q=\left(Q ; z_{1}, z_{2}, z_{3}, z_{4}\right)$ be a quadrilateral with boundary $\partial Q=\cup_{k=1}^{4} \partial Q_{k}$, where all four boundary arcs are assumed to be non-degenerate. Consider the following Dirichlet-Neumann problem already stated in 
TABLE 2.1

The values of moduli of circular quadrilaterals.

\begin{tabular}{c|c|c|c|c}
$\alpha$ & $\mathrm{j}$ & rough accuracy & higher accuracy & $h p$-FEM \\
\hline & 1 & 1.65195637087856 & 1.65195641811156 & 1.65195641811801 \\
$\pi / 4$ & 2 & 1.41312892318176 & 1.41312882432748 & 1.41312882433334 \\
& 3 & 1.23851630005081 & 1.23851628549016 & 1.23851628549600 \\
& 4 & 1.10517568205164 & 1.10517573064876 & 1.10517573065505 \\
& 5 & 1. (sharp value) & 1. (sharp value) & 1.00000000000704 \\
\hline & 1 & 0.98160716203795 & 0.98160730939538 & 0.98160730941547 \\
& 2 & 0.88131392216865 & 0.88131392866493 & 0.88131392869094 \\
$\pi / 5$ & 3 & 0.79679231514866 & 0.79679236427334 & 0.79679236430546 \\
& 4 & 0.72458905475484 & 0.72458889240001 & 0.72458889243949 \\
& 5 & 0.66218846198336 & 0.66218813398119 & 0.66218813402464 \\
\hline \multirow{\pi}{*}{$\pi / 6$} & 1 & 0.69813401618400 & 0.69813355689778 & 0.69813355697485 \\
& 2 & 0.63911291428315 & 0.63911229266297 & 0.63911229274088 \\
& 3 & 0.58614443760266 & 0.58614411420414 & 0.58614411428162 \\
& 4 & 0.53833141064728 & 0.53833144748697 & 0.53833144756331 \\
& 5 & 0.49493995006987 & 0.49493951440663 & 0.49493951447948 \\
\hline \multirow{\pi}{*}{$\pi / 7$} & 1 & 0.54204363753707 & 0.54204377899126 & 0.54204377906567 \\
& 2 & 0.50133118737030 & 0.50133063755764 & 0.50133063763325 \\
& 3 & 0.46350927171723 & 0.46350872114770 & 0.46350872122462 \\
& 4 & 0.42826417448376 & 0.42826373909062 & 0.42826373916846 \\
& 5 & 0.39531876405162 & 0.39531863465020 & 0.39531863472915 \\
\hline \multirow{\pi}{*}{$\pi / 8$} & 1 & 0.44327621319647 & 0.44327582367411 & 0.44327582393810 \\
& 2 & 0.41254694695236 & 0.41254658974644 & 0.41254659003158 \\
& 3 & 0.38338345187308 & 0.38338339855016 & 0.38338339885322 \\
& 4 & 0.35565053319540 & 0.35565066792949 & 0.35565066823961 \\
& 5 & 0.32922105387009 & 0.32922144646084 & 0.32922144678543
\end{tabular}

the introduction:

$$
\left\{\begin{array}{rlrl}
\Delta u & =0, & & \text { on } Q, \\
u & =1, & & \text { on } \partial Q_{1}, \\
u & =0, & & \text { on } \partial Q_{3}, \\
\partial u / \partial n=0, & & \text { on } \partial Q_{2}, \\
\partial u / \partial n=0, & & \text { on } \partial Q_{4} .
\end{array}\right.
$$

Assume that $u$ is the (unique) harmonic solution of the Dirichlet-Neumann problem (3.1). Then the modulus of $Q$ is defined as

$$
\operatorname{Mod}(Q)=\iint_{\Omega}|\nabla u|^{2} d x d y .
$$

Due to (3.2), the modulus of a quadrilateral is the Dirichlet integral, i.e., the $H^{1}$-seminorm of the potential $u$ squared, or, in other words, the energy norm squared, a quantity of interest that is natural in the FEM setting.

3.2. Mesh refinement and exponential convergence. The idea behind the $p$-version is to associate degrees of freedom to topological entities of the mesh in contrast to the classical $h$-version, where this is done with respect to mesh nodes only. The shape functions are based 


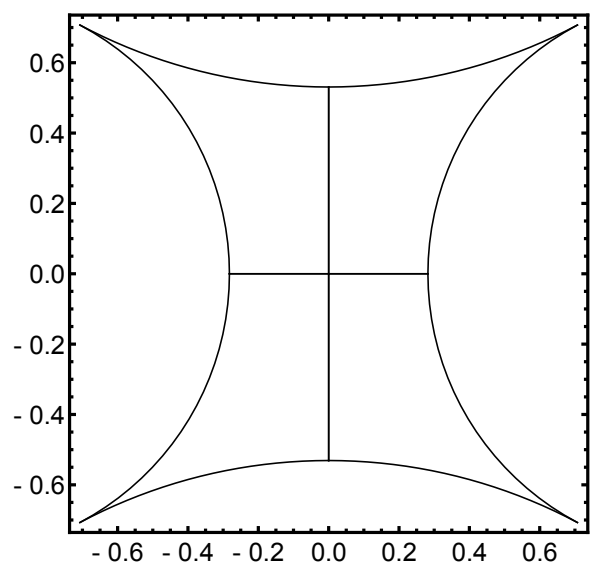

(a) Q1: Mesh; $\alpha=\pi / 4$.

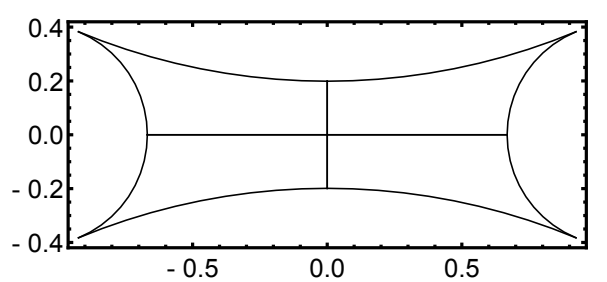

(b) Q2: Mesh; $\alpha=\pi / 8$.

FIGURE 3.1. Circular quadrilaterals. Pure p-version meshes.

on suitable orthogonal polynomials, and their supports reflect the related topological entities, nodes, edges, faces (in 3D), and interiors of the elements. The nodal shape functions induce a partition of unity.

In many problem classes it can be shown that if the mesh is graded appropriately, then the method convergences exponentially in some general norm such as the $H^{1}$-seminorm. Moreover, due to the construction of shape functions, it is natural to have large curved elements in the mesh without a significant increase in the discretization error. Since the number of elements can be kept relatively low given that additional refinement can always be added via the elementwise polynomial degree, a variation in the boundary can be addressed directly at the level of the boundary representation in some exact parametric form.

To fully realize the potential of the $p$-version, one has to grade the meshes properly, and therefore we really use the $h p$-version here. Consider the meshes in Figures 3.2 and 3.1. In Figures 3.2 the basic refinement strategy is illustrated. We start with an initial mesh, where the corners with singularities are isolated, that is, the subsequent refinements of their neighboring elements do not interfere with each other. Then the mesh is refined using successive applications of replacement rules.

In our implementation, the geometry can be described in exact arithmetic, and therefore there are no fixed limits on the number of refinement levels. In the case of graded meshes, one has to resolve the question of how to set the polynomial degrees at every element; indeed, this is a form of refinement of its own. One option in the case of strong singularities is to set the polynomial degree based on the graph distance from the singularity. Alternatively, the degree $p$ can be constant over the whole mesh despite the grading.

3.3. Error estimation. Assuming that the exact capacity is not known, we have two types of error estimates available: the reciprocal estimate and an a posteriori estimate. Naturally, if the exact value is known, we can measure the true error.

3.3.1. Reciprocal error estimate. The first error estimate is rather unusual in the sense that it is based on physics, yet only necessary. For every quadrilateral, the so-called reciprocal relation can be used; see Definition 1. More detailed, from the definition of the modulus via conformal mapping it is clear that the following reciprocal identity holds:

$$
\operatorname{Mod}(Q) \operatorname{Mod}(\tilde{Q})=1 .
$$




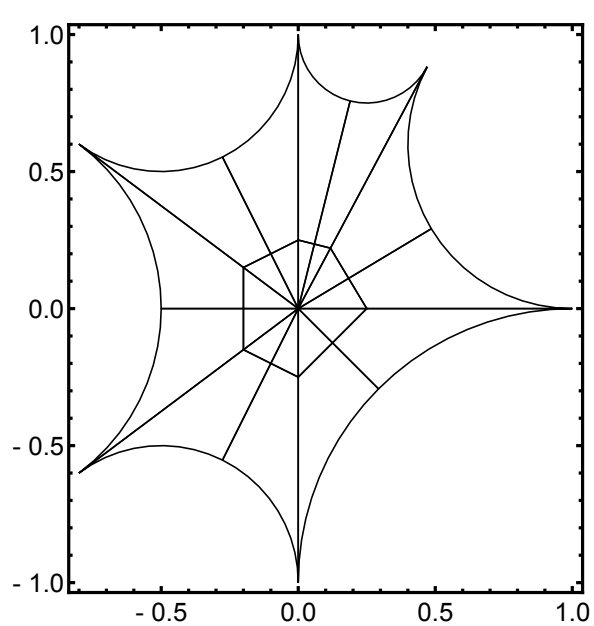

(a) Initial mesh.

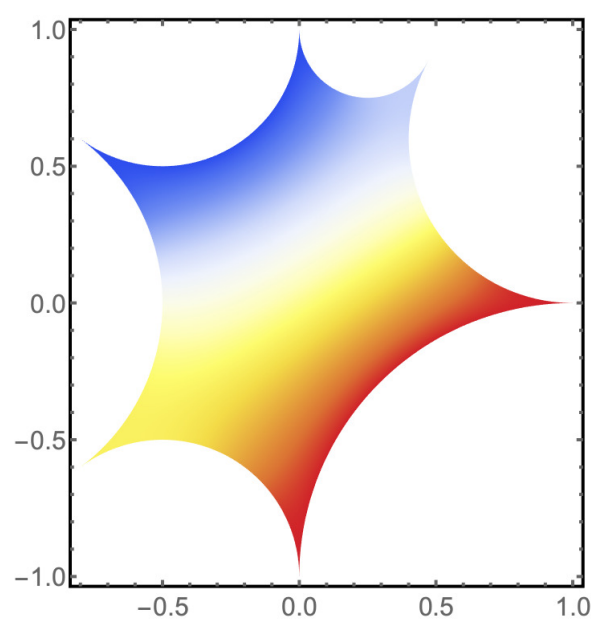

(c) Potential of the problem.

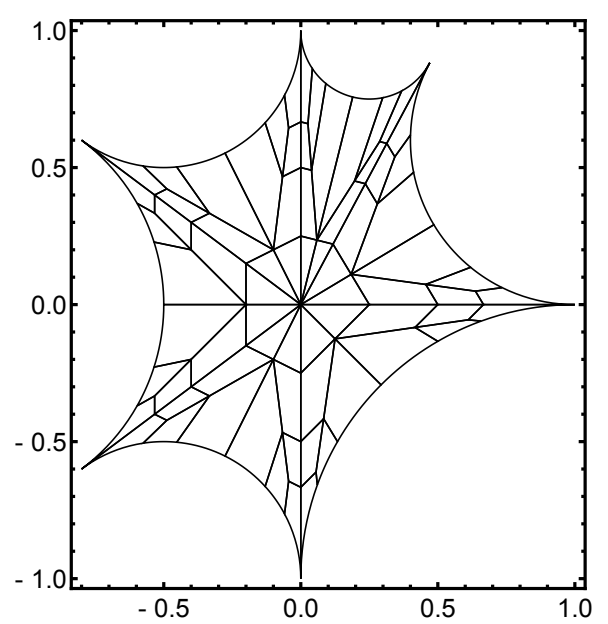

(b) After two levels of refinement.

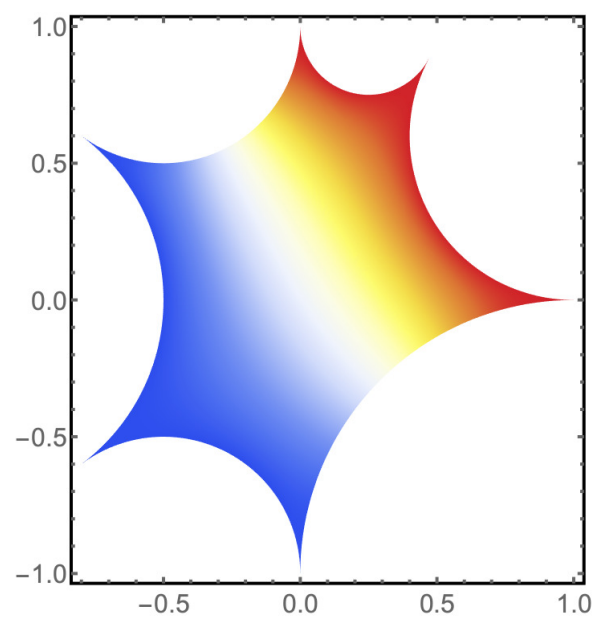

(d) Potential of the conjugate problem.

FIGURE 3.2. Circular hexagon.

Here $\tilde{Q}=\left(Q ; z_{2}, z_{3}, z_{4}, z_{1}\right)$ is called the quadrilateral conjugate to $Q=\left(Q ; z_{1}, z_{2}, z_{3}, z_{4}\right)$. In the numerical context, (3.3) gives us the following error characteristics.

DEFINITION 1 (Reciprocal identity and error). We will call

$$
\varepsilon_{R}=|1-\operatorname{Mod}(Q) \operatorname{Mod}(\tilde{Q})|
$$

the error measure and $\varepsilon_{N}=\left|\left\lceil\log _{10}\left|\varepsilon_{R}\right|\right\rceil\right|$ the related error number.

3.3.2. Auxiliary space error estimate. Consider the abstract problem setting with $u$ as the standard piecewise polynomial finite element space on some discretization $T$ of the computational domain $D$. Assuming that the exact solution $u \in H_{0}^{1}(D)$ has finite energy, we arrive at the following approximation problem: find $\hat{u} \in V$ such that

$$
a(\hat{u}, v)=l(v)(=a(u, v)) \quad \forall v \in V,
$$


TABLE 3.1

Examples: parameters or coordinates used to define the problems. The problem for the hexagon is obtained from Example 2.5 (cf. Figure 2.3(A)) after carrying out the Möbius transformation of Remark 2.6.

(a) Geometry definitions.

\begin{tabular}{ll} 
Example & Parameters or coordinates \\
\hline$Q_{1}$ & $\alpha=\pi / 4, t=\frac{1}{5}(4+\sqrt{2}), s=\frac{1}{23}(20+19 \sqrt{2})$, \\
& $r_{1}=\frac{1}{5} \sqrt{33-12 \sqrt{2}}, r_{2}=\frac{1}{23} \sqrt{777+300 \sqrt{2}}$ \\
$Q_{2}$ & $\alpha=\pi / 8, t=\sec \left(\frac{\pi}{8}\right), s=\csc \left(\frac{\pi}{8}\right)$, \\
& $r_{1}=\tan \left(\frac{\pi}{8}\right), r_{2}=1+\sqrt{2}$ \\
hexagon & $A^{\prime}=-i, B^{\prime}=1, C^{\prime}=\frac{8}{17}+\frac{15}{17} i$, \\
& $D^{\prime}=i, E^{\prime}=-\frac{4}{5}+\frac{3}{5} i, F^{\prime}=-\frac{4}{5}-\frac{3}{5} i$ \\
\hline
\end{tabular}

(b) Problem definitions.

\begin{tabular}{lll} 
Example & Problem & Conjugate \\
\hline hexagon & $Q=\left(Q ; A^{\prime}, B^{\prime}, D^{\prime}, E^{\prime}\right)$ & $\tilde{Q}=\left(Q ; B^{\prime}, D^{\prime}, E^{\prime}, A^{\prime}\right)$
\end{tabular}

where $a(\cdot, \cdot)$ and $l(\cdot)$ are the bilinear form and the load potential, respectively. Additional degrees of freedom can be introduced by enriching the space $V$. This is accomplished via the introduction of an auxiliary subspace or "error space" $W \subset H_{0}^{1}(D)$ such that $V \cap W=\{0\}$. We can then define the error problem: find $\varepsilon \in W$ such that

$$
a(\varepsilon, v)=l(v)-a(\hat{u}, v)(=a(u-\hat{u}, v)) \quad \forall v \in W .
$$

This is simply a projection of the residual to the auxiliary space. In $2 \mathrm{D}$, the space $W$, that is, the additional unknowns, can be associated with element edges and interiors. Thus, for $h p$-methods, this kind of error estimation is natural. The main result for this kind of estimators is the following theorem.

THEOREM 3.1 ([28]). There is a constant $K$ depending only on the dimension $d$, the polynomial degree $p$, the continuity and coercivity constants $C$ and $c$, and the shape-regularity of the triangulation $\mathcal{T}$ such that

$$
\frac{c}{C}\|\varepsilon\|_{1} \leq\|u-\hat{u}\|_{1} \leq K\left(\|\varepsilon\|_{1}+\operatorname{osc}(R, r, \mathcal{T})\right),
$$

where the residual oscillation depends on the volumetric and face residuals $R$ and $r$, and the triangulation $\mathcal{T}$.

The solution $\varepsilon$ of (3.4) is called the error function. It has many useful properties for both theoretical and practical considerations. In particular, the error function can be numerically evaluated and analyzed for any finite element solution. By construction, the error function is identically zero at the mesh points. In the examples below, the space $W$ contains edge shape functions of degree $p+1$ and internal shape functions of degrees $p+1$ and $p+2$. This choice is not arbitrary but based on a careful cost analysis [28].

3.3.3. Examples. The numerical examples are defined in Table 3.1. We consider in detail two circular quadrilaterals and one hexagon. The related results of Table 2.1 above have been obtained with the method discussed here.

In Figures 3.3 and 3.4, different error measures and the related convergence rate in $p$ are shown. In all cases exponential convergence is realized. The estimated rates obtained through 


\section{ETNA}

Kent State University and

Johann Radon Institute (RICAM)

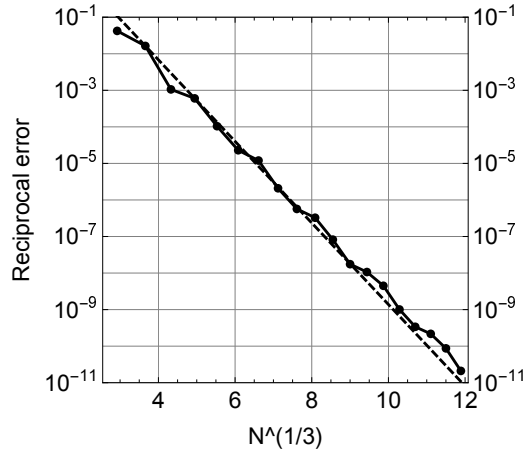

(a) Q1: Reciprocal error; $c=1 / 3$.

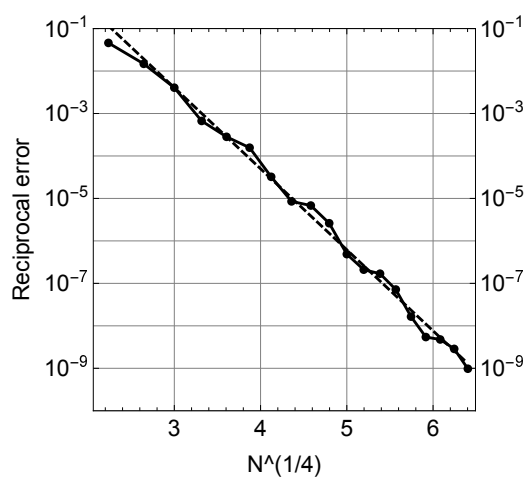

(c) Q2: Reciprocal error; $c=1 / 4$.

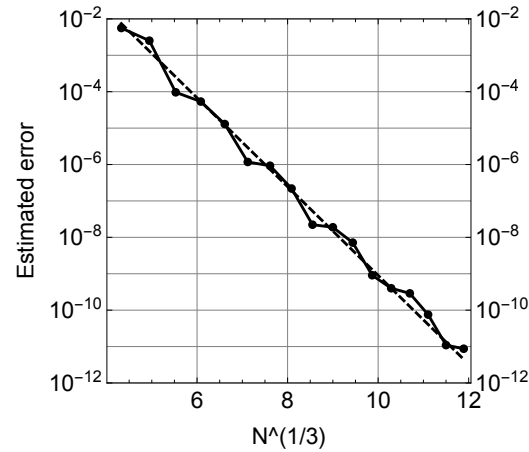

(b) Q1: Estimated error; $c=1 / 3$.

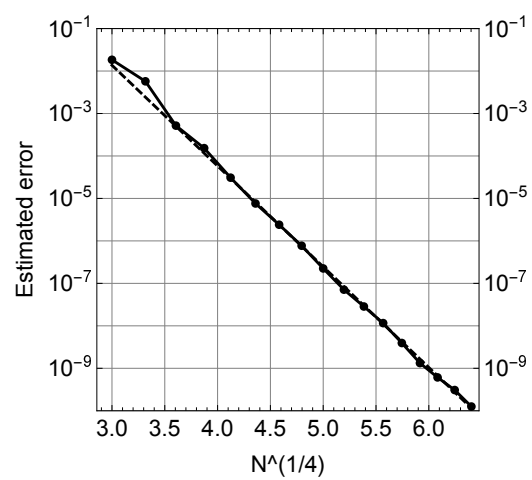

(d) Q2: Estimated error; $c=1 / 4$.

FIGURE 3.3. Circular quadrilaterals. Different types of errors vs. the number of degrees of freedom; log-plots. The solid line with markers represents the observed errors and the dashed line the fitted exponential curve with rate $c$ indicated in the caption.

nonlinear fitting are also indicated (with dashed lines), and the parameters used are given in Table 3.2. One has to remember that such fits are notoriously sensitive to selected points, and thus the rates given here should be taken as possible rates rather than the definitive ones. We have used a visualization technique where the scaling is selected such that the observed graph appears linear.

For the two quadrilaterals $Q_{1}$ and $Q_{2}$, the results are very good indeed. In fact, the estimated rate for $Q_{1}$ is the theoretically optimal one in terms of the number of degrees of freedom, of course with a large constant [40]. The reason behind such a spectacular accuracy is that the underlying mapping of the curved elements, the blending function mapping, is exact for circular boundary segments. From the point of view of the method, the corner singularity is practically removed by the mapping. For $Q_{2}$ with the smaller aspect ratio, at higher polynomial orders, there is a degradation of the convergence rate in comparison to the symmetric domain of $Q_{1}$, and indeed, the selected scaling cannot remain the same as for $Q_{1}$. Notice that the exceedingly large constant $a_{1}$ of the estimated error is due to the non-trivial oscillation in the estimate, and the asymptotic convergence is reached only at high values of $p$. Due to symmetry, $\operatorname{Mod}\left(Q_{1}\right)=1$. We have not shown the error in capacity in this case. This is done in the following case, however. 


\section{ETNA}

Kent State University and

Johann Radon Institute (RICAM)

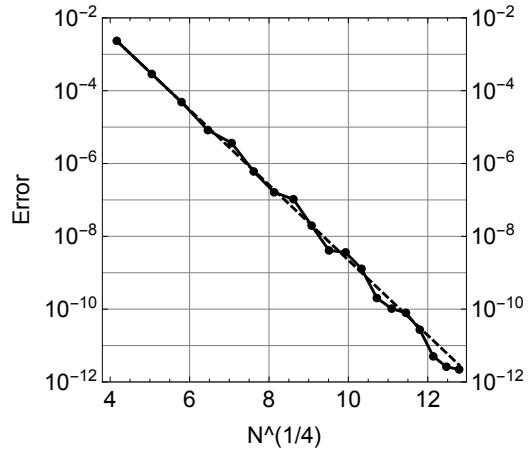

(a) Error in capacity; $c=1 / 4$.

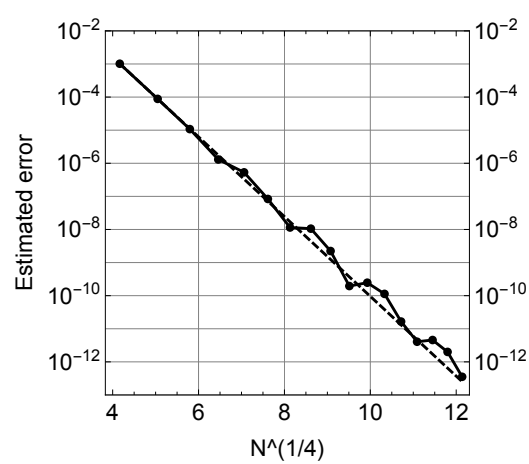

(c) Estimated error; $c=1 / 4$.

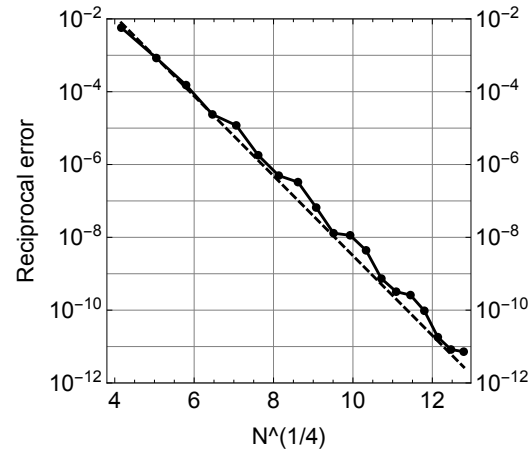

(b) Reciprocal error; $c=1 / 4$.

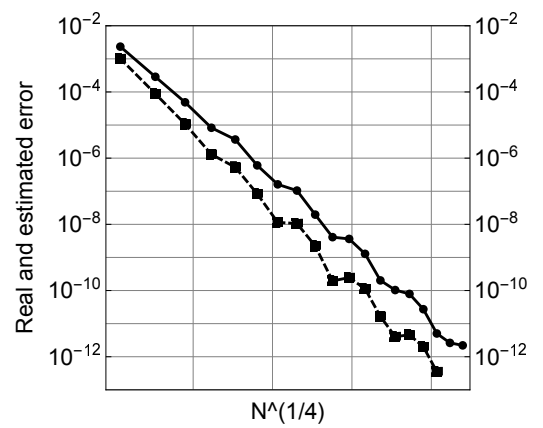

(d) Comparison of the real and estimated errors.

FIGURE 3.4. Circular hexagon. Different types of errors vs. the number of degrees of freedom; log-plots. The solid line with markers represents the observed errors and the dashed line the fitted exponential curve with rate $c$ indicated in the caption. In the comparison graph the estimated error is smaller with asymptotic effectivity of 1/10.

For the circular hexagon, the geometric meaning of the domain and its conjugate is illustrated in Figures 3.2c and 3.2d. In this case, the exact modulus is also known,

$$
\operatorname{Mod}(Q)=\tau(\sqrt{2}) / 2=\frac{K(1 / \sqrt{1+\sqrt{2}})}{K(\sqrt{\sqrt{2} /(1+\sqrt{2})})} \approx 0.92401502327430725964 \ldots,
$$

where $K(r)$ is the complete elliptic integral. The method is indeed very accurate, and the observed rate is within the expected range. As is often the case, the auxiliary space estimate trails the true error, yet the effectivity is still over $1 / 10$ as $p$ increases. Due to the construction of the auxiliary space, the estimate is computed at a lower polynomial order.

In Table 3.2 the error numbers for the reciprocal error estimates are also reported for the highest polynomial order. These results are aligned with those reported for similar problems before; see [30].

REMARK 3.2 (On the computational complexity of the error estimates). The two error estimates do not differ in their computational complexity in any significant way. Although the reciprocal error estimate requires the solution of two problems and the auxiliary space estimate is for one problem only, the cost of the numerical integration (always an issue in high-order methods) is roughly the same, and the two solution steps for the reciprocal error estimate can share the Cholesky factorization of the interior degrees of freedom. 


\section{ETNA}

Kent State University and

Johann Radon Institute (RICAM)

TABLE 3.2

Parameters of the nonlinear fit $a_{1} \exp \left(a_{2} N^{c}\right)$, where $N$ is the number of degrees of freedom, and the error numbers for the reciprocal errors.

\begin{tabular}{llrrrrr} 
Example & Error Type & $a_{1}$ & $a_{2}$ & $c$ & $N(p=20)$ & Error Number \\
\hline$Q_{1}$ & Estimated & 1397 & -2.8 & $1 / 3$ & 1681 & \\
& Reciprocal & 198 & -2.6 & $1 / 3$ & 1681 & 10 \\
$Q_{2}$ & Estimated & 179191 & -5.5 & $1 / 4$ & 1681 & 9 \\
\multirow{4}{*}{ hexagon } & Reciprocal & 2154 & -4.4 & $1 / 4$ & 1681 & \\
& True & 47 & -2.4 & $1 / 4$ & 26761 & 11
\end{tabular}

REMARK 3.3 (On the performance comparison between the Schwarz ODE and the $h p$-FEM). As mentioned above, the quadrilateral example is particularly well-suited to the $h p$-FEM. This makes it somewhat awkward to compare the computational efficiency of the two numerical approaches presented in this paper. Of course, one should also take into account the time spent in defining the computational domain. This is very difficult to measure, however. The Schwarz ODE routine and the $h p$-solver have comparable performance when the former is run using standard precision. Due to the implementation of the ODE solver, the higher accuracy is obtained only by changing the floating-point representation, which leads to higher run times. On the other hand, the Schwarz ODE has an almost uniform runtime characteristics over all circular quadrilaterals, and it is likely that replacing the general ODE solver routines with problem specific ones will lead to significant improvements in run times. The $h p$-FEM requires more resources if the discretization includes more elements. With the current $h p$ implementation, the non-graded discretization of the $n$-gon (Figure 3.2a) took three times longer than the corresponding quadrilaterals; see Figure 3.1.

4. Conclusions. Here, moduli of planar circular quadrilaterals symmetric with respect to both coordinate axes have been investigated. The computation of moduli of planar domains with cusps is difficult and requires either a customized analytic algorithm or a general method with sufficient flexibility. The Schwarz ODE introduced here, an analytic method to determine a conformal mapping of the unit disk onto a given circular quadrilateral, belongs to the first category. $h p$-FEM on the other hand provides a framework for highly efficient numerical PDE solvers. We have shown that these two different approaches provide results agreeing with high accuracy over two sets of parametrized examples. The results have been verified using a problem with analytic solution and, in the case of the $h p$-FEM, by two different error estimates. From the point-of-view of computational complexity, the choice of the preferred method depends on many factors, and it is not possible to arrive at a general recommendation.

One interesting application is the quadrature design for finite elements on curvilinear polygons or $n$-gons. Constructing quadrature rules for such elements is a challenge, and employing conformal mappings is an intriguing option yet to be fully examined.

Appendix A. Reference implementations. The programs used to compute the results of Table 2.1 are available at https://github.com/hhakula/hnv, and Version 1.0, used in this paper, is archived at DOI: $10.5281 /$ zenodo. 4718350.

The Schwarz ODE code is also listed below. The expected output of the program is 
LISTING 1

Schwarz $O D E$.

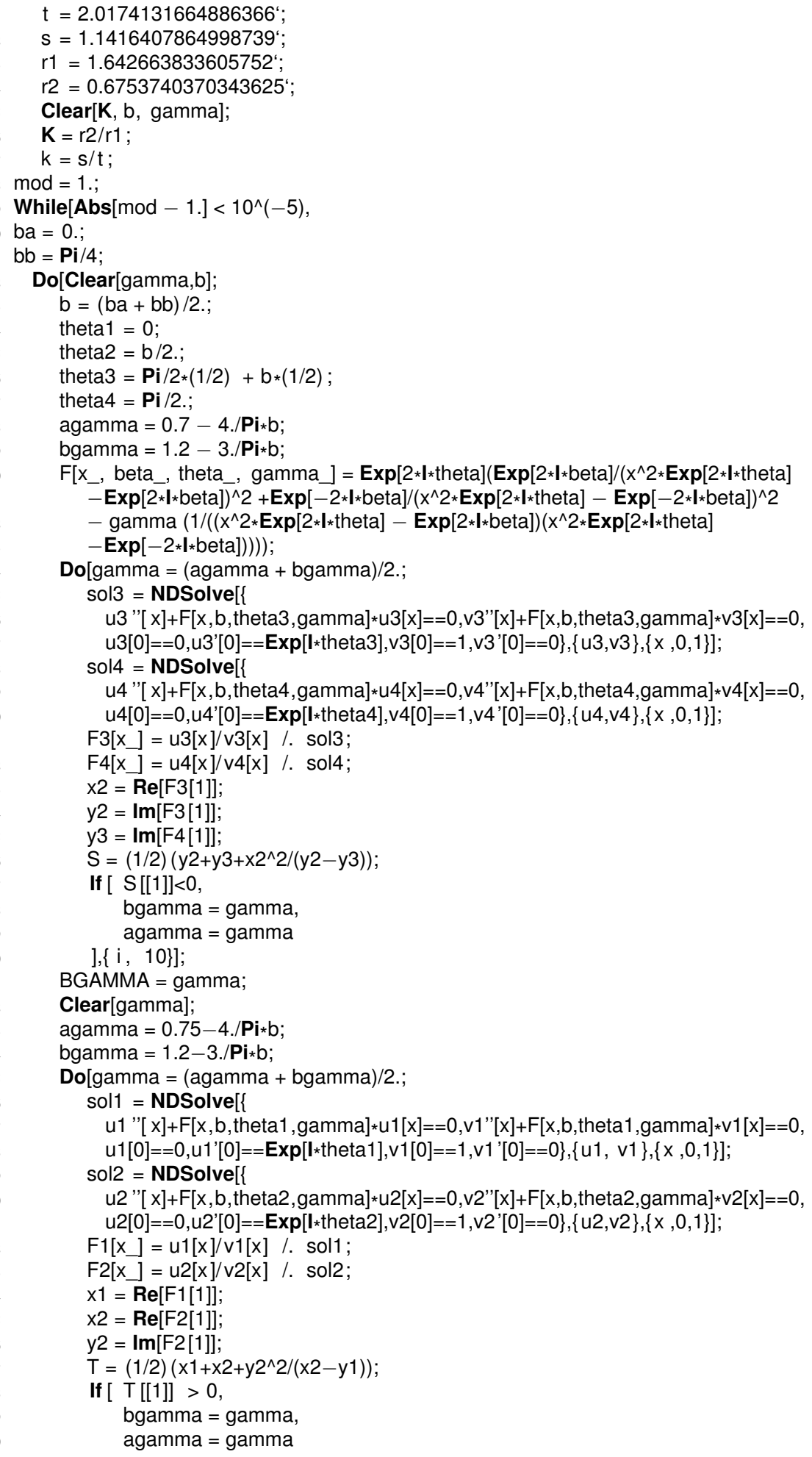


], $\{\mathrm{i}, 10\}]$

AGAMMA = gamma;

Clear[gamma, $x 1, x 2, y 2, x 3, y 3, y 4]$;

Do[gamma $=($ AGAMMA + BGAMMA $) / 2$;

sol1 $=$ NDSolve $[\{$

u1 " $[x]+F[x, b$, theta 1, gamma $] * u 1[x]==0, v 1$ " $[x]+F[x, b$, theta 1, gamma $] * v 1[x]==0$, $\mathrm{u} 1[0]==0, \mathrm{u} 1^{1}[0]==\operatorname{Exp}[1 \times$ theta1 $\left.], \mathrm{v} 1[0]==1, \mathrm{v} 1{ }^{\prime}[0]==0\right\},\{\mathrm{u} 1, \mathrm{v} 1\},\{\mathrm{x}, 0,1\}$,

PrecisionGoal->15];

sol2 $=$ NDSolve $[\{$

u2 " $[x]+F[x, b$, theta2, gamma $] * u 2[x]==0, v 2$ " $[x]+F[x, b$, theta2, gamma $] * v 2[x]==0$, $\mathrm{u} 2[0]==0, \mathrm{u} 2^{\prime}[0]==\operatorname{Exp}[1 *$ theta2 $\left.], \mathrm{v} 2[0]==1, \mathrm{v} 2^{\prime}[0]==0\right\},\{\mathrm{u} 2, \mathrm{v} 2\},\{x, 0,1\}$,

PrecisionGoal $->15]$;

sol3 $=$ NDSolve $[\{$

u3 " $[\mathrm{x}]+\mathrm{F}[\mathrm{x}, \mathrm{b}$, theta3, gamma $] * \mathrm{u} 3[\mathrm{x}]==0, \mathrm{v} 3^{\prime \prime}[\mathrm{x}]+\mathrm{F}[\mathrm{x}, \mathrm{b}$, theta3, gamma $] * \mathrm{v} 3[\mathrm{x}]==0$, $\mathrm{u} 3[0]==0, \mathrm{u} 3^{\prime}[0]==\operatorname{Exp}[1 \times$ theta3 $\left.], \mathrm{v} 3[0]==1, \mathrm{v}^{\prime}[0]==0\right\},\{\mathrm{u} 3, \mathrm{v} 3\},\{\mathrm{x}, 0,1\}$,

PrecisionGoal->15];

sol4 $=$ NDSolve $[\{$

$\mathrm{u} 4$ " $[\mathrm{x}]+\mathrm{F}[\mathrm{x}, \mathrm{b}$, theta 4, gamma $] * \mathrm{u} 4[\mathrm{x}]==0, \mathrm{v} 4$ ' $[\mathrm{x}]+\mathrm{F}[\mathrm{x}, \mathrm{b}$, theta 4, gamma $] * \mathrm{v} 4[\mathrm{x}]==0$, $u 4[0]==0, u 4^{\prime}[0]==\operatorname{Exp}[\mid *$ theta 4$\left.], v 4[0]==1, v 4^{\prime}[0]==0\right\},\{u 4, v 4\},\{x, 0,1\}$,

PrecisionGoal $->15]$;

$\mathrm{F} 1[\mathrm{x}]=\mathrm{u} 1[\mathrm{x}] / \mathrm{v} 1[\mathrm{x}] /$. sol1;

$\mathrm{F} 2[\mathrm{x}]=\mathrm{u} 2[\mathrm{x}] / \mathrm{v} 2[\mathrm{x}] /$. sol 2 ;

$F 3[x]=u 3[x] / v 3[x] /$. sol3;

$\mathrm{F} 4[\mathrm{x}]=\mathrm{u} 4[\mathrm{x}] / \mathrm{v} 4[\mathrm{x}] /$. sol 4 ;

$\mathrm{x} 1=\operatorname{Re}[\mathrm{F} 1[1]]$

$x 2=\operatorname{Re}[F 2[1]]$

$\mathrm{y} 2=\operatorname{Im}[\mathrm{F} 2[1]]$

$T=(1 / 2)\left(x 1+x 2+y 2^{\wedge} 2 /(x 2-x 1)\right)$

$\mathrm{R} 1=\operatorname{Abs}[\mathrm{x} 1-\mathrm{T}]$;

$\mathrm{x} 3=\operatorname{Re}[\mathrm{F} 3[1]]$

$\mathrm{y} 3=\operatorname{Im}[\mathrm{F} 3[1]]$

$\mathrm{y} 4=\operatorname{lm}[\mathrm{F} 4[1]]$

$S=(1 / 2)\left(y 3+y 4+x 3^{\wedge} 2 /(y 3-y 4)\right) ;$

$\mathrm{R} 2=\operatorname{Abs}[\mathrm{y} 4-\mathrm{S}]$;

If $[k * T[[1]]<S[[1]]$,

BGAMMA = gamma,

AGAMMA $=$ gamma

],\{ i ,25\}];

If $[R 2[[1]] / R 1[[1]]<K$,

$b b=b$,

$\mathrm{ba}=\mathrm{b}$

]$,\{n, 25\}]$;

$\mathrm{m}=(\operatorname{Tan}[\mathrm{b} / 2])^{\wedge} 4$;

$\bmod =2 \star$ EllipticK $[\mathrm{m}] /$ EllipticK $[1-\mathrm{m}]$;

If $\left[\mathbf{A b s}[\bmod -1]<.10^{\wedge}(-5)\right.$,

$K=r 1 / r 2$

];

If $\left[\operatorname{Abs}[\bmod -1]<.10^{\wedge}(-5)\right.$,

$\mathrm{k}=\mathrm{t} / \mathrm{s}$

];

]; ( * End top While *)

If $[\mathbf{K}==\mathrm{r} 2 / \mathrm{r} 1$,

b1 $=b$,

];

$\mathrm{b} 1=\mathbf{P i} / 2-\mathrm{b}$

$\mathrm{m} 1=(\operatorname{Tan}[\mathrm{b} 1 / 2])^{\wedge} 4 ;$

$\operatorname{modQ}=2 \star$ EllipticK[m1]/EllipticK[1-m1];

$\{b 1$, gamma, modQ\} 


\section{REFERENCES}

[1] L. V. Ahlfors, Conformal Invariants: Topics in Geometric Function Theory, McGraw-Hill, New York, 1973.

[2] L. V. Ahlfors AND A. Beurling, Conformal invariants and function-theoretic null sets, Acta Math., 83 (1950), pp. 101-129.

[3] N. I. AKHIEZER, Elements of the Theory of Elliptic Functions, Translations of Mathematical Monographs 79, American Mathematical Society, Providence, 1990.

[4] I. A. AleKsandrov, Parametric continuations in the theory of univalent functions (in Russian), Nauka, Moscow, 1976.

[5] A. Anand, J. S. Ovall, S. E. Reynolds, AND S. Weisser, Trefftz finite elements on curvilinear polygons, SIAM J. Sci. Comput., 42 (2020), pp. A1289-A1316.

[6] G. D. Anderson, M. K. Vamanamurthy, And M. Vuorinen, Conformal Invariants, Inequalities and Quasiconformal maps, Wiley, New York, 1997.

[7] I. BABUŠKA, X. HUANG, AND R. LIPTON, Machine computation using the exponentially convergent multiscale spectral generalized finite element method, ESAIM Math. Model. Numer. Anal., 48 (2014), pp. 493-515.

[8] I. BABUŠKA AND M. SURI, The $p$ and $h-p$ versions of the finite element method, basic principles and properties, SIAM Rev., 36 (1994), pp. 578-632.

[9] B. G. BAǏBARIN, On a numerical method for determining the parameters of the Schwarz derivative for a function conformally mapping the half-plane onto circular domain., Trudy Tomsk. Gos. Univ. Ser. Meh.-Mat., 189 (1966), pp. 123-136.

[10] U. BAUER AND W. LAUF, Conformal mapping onto a doubly connected circular arc polygonal domain, Comput. Methods Funct. Theory, 19 (2019), pp. 77-96.

[11] A. F. Beardon, Curvature, circles, and conformal maps, Amer. Math. Monthly, 94 (1987), pp. 48-53.

[12] L. Beirão da Veiga, F. Brezzi, A. Cangiani, G. Manzini, L. D. Marini, and A. Russo, Basic principles of virtual element methods, Math. Models Methods Appl. Sci., 23 (2013), pp. 199-214.

[13] E. N. BERESLAVSKIII AND L.M. DuDINA, On the flow of groundwater to an imperfect gallery in the presence of evaporation with a free surface, Math. Model., 20 (2018), pp. 99-109.

[14] P. BJøRSTAD AND E. Grosse, Conformal mapping of circular arc polygons. SIAM J. Sci. Statist. Comput., 8 (1987), pp. 19-32.

[15] P. R. BRown, Mapping onto circular arc polygons, Complex Var. Theory Appl., 50 (2005), pp. 131-154.

[16] _ An investigation of a two parameter problem for conformal maps onto circular arc quadrilaterals, Complex Var. Elliptic Equ., 53 (2008), pp. 23-51.

[17] P. R. BROWN AND R. M. PORTER, Conformal mapping of circular quadrilaterals and Weierstrass elliptic functions, Comput. Methods Funct. Theory, 11 (2011), pp. 463-486.

[18] E. Burman, S. Claus, P. Hansbo, M. G. Larson, and A. Massing, CutFEM: discretizing geometry and partial differential equations, Internat. J. Numer. Methods Engrg., 104 (2015), pp. 472-501.

[19] L. I. ChIBRIKova, Selected Chapters in the Analytic Theory of Ordinary Differential Equations (in Russian), Kazanskiǔ Fond "Matematika", Kazan, 1996.

[20] YU. V. CHISTYAKOV, On a method of approximate computation of a function mapping conformally the circle onto domain bounded by circular arcs and straight line segments, Uch. Zap. Tomsk. Univ., 14 (1960), pp. 143-151.

[21] D. G. CRowdy, A. S. FoKas, AND C. C GReEn, Conformal mappings to multiply connected polycircular arc domains, Comput. Methods Funct. Theory, 11 (2011), pp. 685-706.

[22] T. A. Driscoll And L. N. Trefethen, Schwarz-Christoffel Mapping, Cambridge University Press, Cambridge, 2002.

[23] V. N. Dubinin, Condenser Capacities and Symmetrization in Geometric Function Theory, Springer, Basel, 2014

[24] V. V. Golubev, Lectures on Analytical Theory of Differential Equations (in Russian), Gostekhizdat, Moscow, 1950.

[25] G. M. Goluzin, Geometric Theory of Functions of a Complex Variable, Translations of Mathematical Monographs 26, American Mathematical Society, Providence, 1969.

[26] A. Gopal And L. N. TRefethen, Solving Laplace problems with corner singularities via rational functions, SIAM J. Numer. Anal., 57 (2019), pp. 2074-2094.

[27] - New Laplace and Helmholtz solvers, Proc. Nat. Acad. Sci. USA, 116 (2019), pp. 10223-10225.

[28] H. HakUla, M. NeIlan, AND J. Ovall, A posteriori estimates using auxiliary subspace techniques, J. Sci. Comput., 72 (2017), pp. 97-127.

[29] H. HAKUla, A. RASila, AND M. VuORINEN, On moduli of rings and quadrilaterals: algorithms and experiments, SIAM J. Sci. Comput., 33 (2011), pp. 279-302.

[30] - Conformal modulus and planar domains with strong singularities and cusps, Electron. Trans. Numer. Anal., 48 (2018), pp. 462-478.

http://etna.ricam.oeaw.ac.at/vol.48.2018/pp462-478.dir/pp462-478.pdf 
[31] H. Hakula And T. TuOminen, Mathematica implementation of the high order finite element method applied to eigenproblems, Computing, 95 (2013), pp. S277-S301.

[32] P. HaRiRI, R. KlÉn, AND M. VuORInen, Conformally Invariant Metrics and Quasiconformal Mappings, Springer, Cham, 2020.

[33] I. A. Kolesnikov, On the problem of determining parameters in the Schwarz equation, Probl. Anal. Issues Anal., 7 (2018), pp. 50-62.

[34] V. V. KRAVChenKo And R. M. Porter, Conformal mapping of right circular quadrilaterals, Complex Var. Elliptic Equ., 56 (2010), pp. 399-415.

[35] R. KÜHNAU (ed.), Handbook of Complex Analysis: Geometric Function Theory. Vol. 2, Elsevier, Amsterdam, 2005.

[36] M. M. S. NASSER, PlgCirMap: A MATLAB toolbox for computing conformal mappings from polygonal multiply connected domains onto circular domains, SoftwareX, 11 (2020), Art. No. 100464, 7 pages.

[37] M. M. S. NASSER AND M. VuORInen, Computation of conformal invariants, Appl. Math. Comput., 389 (2021), 125617 (24 pages)

[38] G. Polya AND G. SZEGÖ, Isoperimetric Inequalities in Mathematical Physics, Princeton University Press, Princeton, 1951.

[39] R. M. PORTER, Numerical calculation of conformal mapping to a disk minus finitely many horocycles, Comput. Methods Funct. Theory, 5 (2005), pp. 471-488.

[40] C. SCHWAB, $p$ - and hp-Finite Element Methods, Oxford University Press, New York, 1998.

[41] L. N. TREFETHEN, Numerical conformal mapping with rational functions, Comput. Methods Funct. Theory, 20 (2020), pp. 369-387.

[42] A. R. Tsitskishvili, On filtration in dams with slopping backs (in Russian), Trudy Tbiliss. Mat. Inst. Razmadze, 11 (1976), pp. 94-104

[43] _ Effective Methods for Solving the Problems of Conformal Mapping and the Theory of Filtration (in Russian), Diss. Doct. Phys.-Math. Sci., Moscow, 1981

[44] - General solution of differential Schwartz equation for conformally mapping functions of circular polugons, their connection with boundary value problems of filtration and of axially symmetric flows, Proc. of A. Razmadze Math. Inst., 153 (2010), pp. 1-148.

[45] W. von Koppenfels and F. Stallmann, Praxis der Konformen Abbildung (in German), Springer, Berlin, 1959. 